\title{
On the Efficiency of Field Star Capture by Star Clusters
}

\author{
S. Mieske ${ }^{1}$ and H. Baumgardt ${ }^{2}$ \\ ${ }^{1}$ European Southern Observatory, Karl-Schwarzschild-Str.2, 85748 Garching b. München, \\ Germany \\ email: smieske@eso.org,mhilker@eso.org \\ ${ }^{2}$ Argelander Institut für Astronomie, Auf dem Hügel 71, 53121 Bonn, Germany \\ email: holger@astro.uni-bonn.de
}

\begin{abstract}
An exciting recent finding regarding scaling relations among globular clusters is the so-called 'blue tilt': clusters of the blue sub-population follow a trend of redder colour with increasing luminosity. In this contribution we estimate by means of collisional $N$-body simulations to which extent this trend can be explained by field star capture occurring over a Hubble time. We investigate star clusters with $10^{3}$ to $10^{6}$ stars. We find that the ratio between captured field stars and total number of clusters stars is very low $\left(\lesssim 10^{-4}\right)$, even for co-rotation of the star cluster in a cold disk. This holds for star clusters in the mass range of both open clusters and globular clusters. Therefore, field star capture is not a probable mechanism for creating the colour-magnitude trend of metal-poor globular clusters.
\end{abstract}

Keywords. globular clusters: general, open clusters and associations: general, stars: kinematics, galaxies: kinematics and dynamics

\section{Introduction}

An exciting recent finding regarding scaling relations among globular clusters is the so-called 'blue tilt': the colours of individual globular clusters (GCs) of the blue subpopulation are correlated with their luminosities such that brighter globulars are redder (Harris et al. 2006, Mieske et al. 2006, Strader et al. 2006, Spitler et al. 2006, Cantiello et al. 2007). Various mechanisms have been discussed that may offer ways towards explaining the trend, like self-enrichment (Strader et al. 2006) or "sample contamination" by stripped nuclei of dwarf galaxies (Harris et al. 2006, Bekki et al. 2007). In Mieske et al. (2006) we indicate that also the capture of field stars in giant elliptical galaxies can in principle cause such colour-magnitude trends and its dependence on galactocentric distance as found by Mieske et al. (2006). This is because the field star population is much redder than the blue globular clusters.

\section{Results and conclusions}

Here, we present results of collisional $N$-body simulations made to quantify the amount of field star capture occurring over a Hubble time to star clusters with $10^{3}$ to $10^{6}$ stars (see also Mieske \& Baumgardt 2007). In the simulations we follow the orbits of field stars passing through a star cluster and calculate the energy change that the field stars experience due to gravitational interaction with cluster stars during one passage through the cluster. The capture condition is that their total energy after the passage is smaller than the gravitational potential at the cluster's tidal radius (see Fig. 1). By folding this with the fly-by rates of field stars with an assumed space density as in the solar 

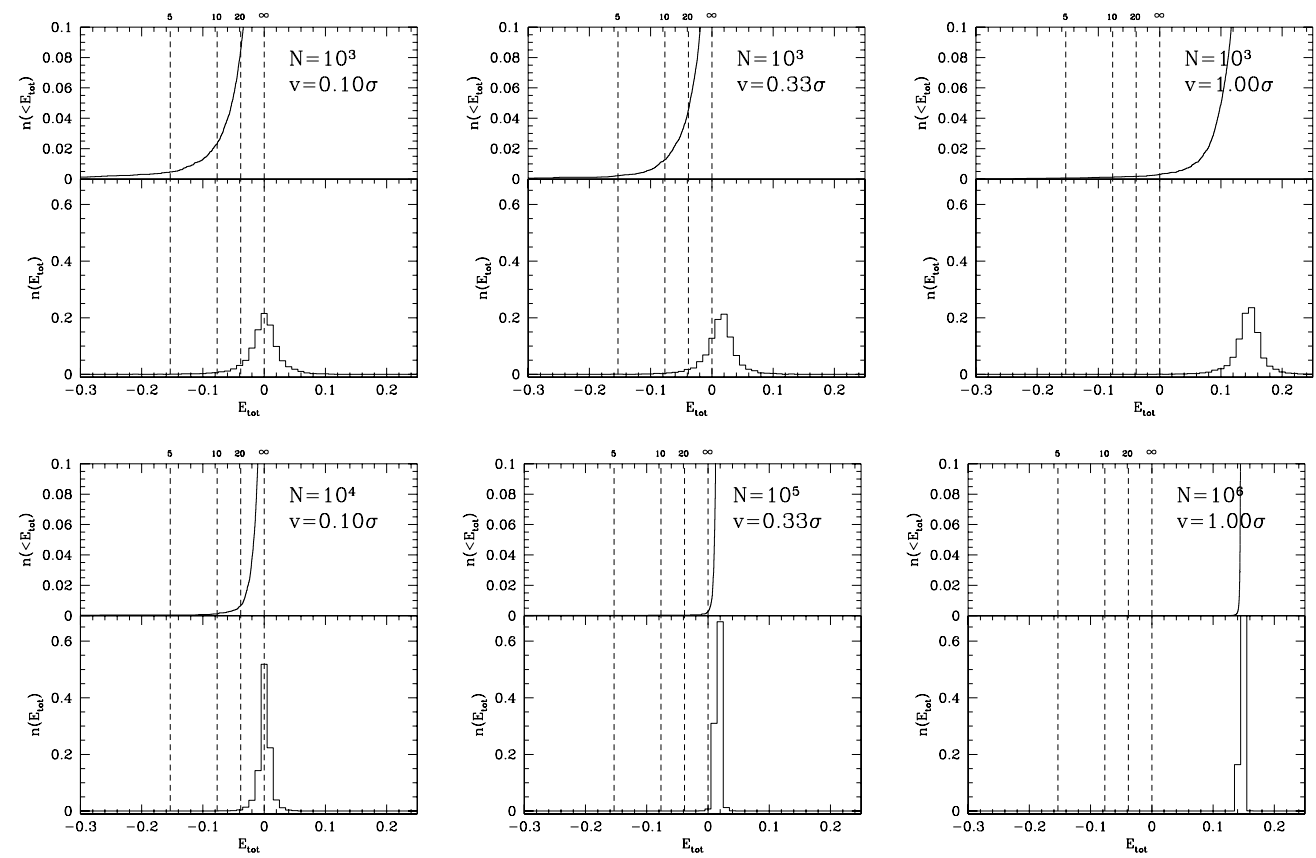

Figure 1. Distribution of total energy $\mathrm{E}_{t o t}$ of field stars after interaction with the cluster stars. The three plots refer to different number of stars in the star clusters, and different assumed initial velocity $v_{i n i}$ of the field stars in units of the star cluster velocity dispersion $\sigma$. Top row, from left to right: star cluster with $N=10^{3}$ stars and field star initial velocities $v_{\text {in } i}=0.1,0.33$ and $1.0 \sigma$. Bottom row, from left to right: star clusters with $N=10^{4}, 10^{5}$ and $10^{6}$ stars with the indicated $v_{i n i}$. The lower part of each plot gives the energy histogram, the upper part gives the cumulative energy distribution. The requirement for field star capture is $E_{t o t}<\frac{-G M_{c}}{r_{\text {tid }}}$, with $r_{\text {tid }}$ being the tidal radius of the cluster in the gravitational field of the host galaxy. The vertical dashed lines indicate different assumed ratios of $\frac{r_{\text {tid }}}{r_{h}}$, where $r_{h}$ is the cluster's half-mass radius. The probability of field star capture is non-negligible only for the case of a low mass cluster and low relative velocity. However, for the given example of $N=10^{3}$ stars, $v_{i n i}=0.1 \sigma$ corresponds to $0.04 \mathrm{~km} / \mathrm{s}$, assuming an average cluster star mass of 0.5 solar masses. The extremely low number of field stars that have such low relative velocities makes the capture rate negligible.

neighbourhood and a range of velocity dispersions $\sigma$ (15 to $485 \mathrm{~km} / \mathrm{s}$ ), we derive estimates on the mass fraction of captured field stars as a function of environment.

From this we find that integrated over a Hubble time, the ratio between captured field stars and total number of clusters stars is very low $\left(\lesssim 10^{-4}\right)$, even for the smallest field star velocity dispersion $\sigma=15 \mathrm{~km} / \mathrm{s}$. This holds for star clusters in the mass range of both open clusters and globular clusters. Field star capture is therefore not a probable mechanism for creating the 'blue tilt'.

\section{References}

Bekki, K., Yahagi, H., \& Forbes, D. A. 2007, MNRAS, 377, 215

Cantiello, M., Blakeslee, J. P., \& Raimondo, G. 2007, astro-ph/0706.3943

Harris, W. E. et al. 2006, ApJ, 636, 90

Mieske, S. et al. 2006, ApJ, 653, 193

Mieske, S. \& Baumgardt, H. 2007, A\&A in press, arXiv:0709.1328

Spitler, L. R. et al. 2006, AJ, 132, 1593

Strader, J., Brodie, J. P., Spitler, L., \& Beasley, M. A. 2006, AJ, 132, 2333 